\title{
Mechanostimulation Promotes Nuclear and Epigenetic Changes in Oligodendrocytes
}

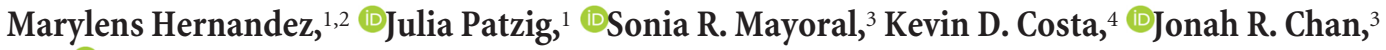 \\ and Patrizia Casaccia ${ }^{1,5}$ \\ ${ }^{1}$ Department of Neuroscience, ${ }^{2}$ Graduate School of Biological Sciences, ${ }^{4}$ Cardiovascular Research Center, and ${ }^{5}$ Department of Genomics and Multiscale \\ Biology, Icahn School of Medicine at Mount Sinai, New York, New York 10029-5674, and ${ }^{3}$ Department of Neurology and Program in Neurosciences, \\ University of California, San Francisco, San Francisco, California 94145-0339
}

Oligodendrocyte progenitors respond to biophysical or mechanical signals, and it has been reported that mechanostimulation modulates cell proliferation, migration, and differentiation. Here we report the effect of three mechanical stimuli on mouse oligodendrocyte progenitor differentiation and identify the molecular components of the linker of nucleoskeleton and cytoskeleton (LINC) complex (i.e., SYNE1) as transducers of mechanical signals to the nucleus, where they modulate the deposition of repressive histone marks and heterochromatin formation. The expression levels of LINC components increased during progenitor differentiation and silencing the Syne1 gene resulted in aberrant histone marks deposition, chromatin reorganization and impaired myelination. We conclude that spatial constraints, via the actin cytoskeleton and LINC complex, mediate nuclear changes in oligodendrocyte progenitors that favor a default pathway of differentiation.

Key words: chromatin; histone; cytoskeleton; myelin; nucleus

\section{Significance Statement}

It is recognized that oligodendrocyte progenitors are mechanosensitive cells. However, the molecular mechanisms translating mechanical stimuli into oligodendrocyte differentiation remain elusive. This study identifies components of the mechanotransduction pathway in the oligodendrocyte lineage.

\section{Introduction}

Myelin is critical for the proper function of the CNS as it insulates axons, allowing rapid synaptic transmission and providing trophic support to neurons (Rinholm et al., 2011; Fünfschilling et al., 2012; Lee et al., 2012). Myelinating oligodendrocytes differentiate in response to soluble chemical signals (Hart et al., 1989; Barres et al., 1994; Gao et al., 1998; Pombo et al., 1999; Lu et al., 2000; Nery et al., 2001; Samanta and Kessler, 2004; Fortin et al., 2005; Zhou and Armstrong, 2007; Furusho et al., 2011; Dugas et al., 2012; Wu et al., 2012) as well as physical perturbations (Rosenberg et al., 2008; Jagielska et al., 2012), but the pathways

\footnotetext{
Received July 30, 2015; revised Nov. 13, 2015; accepted Dec. 5, 2015.

Author contributions: K.D.C., J.R.C., and P.C. designed research; M.H., J.P., and S.R.M. performed research; K.D.C. contributed unpublished reagents/analytic tools; M.H., J.P., K.D.C., J.R.C., and P.C. analyzed data; M.H., J.P., and P.C. wrote the paper.

This work was supported by Grant F31NS083344 to M.H. and Grants R01NS52738 and R37NS42925 to P.C. from the National Institute of Neurological Disorders and Stroke at the National Institute of Health. We thank Dr. M. Wu for initial experiments.

The authors declare no competing financial interests.

Correspondence should be addressed to Patrizia Casaccia, Icahn School of Medicine at Mount Sinai, One Gustave Levy Place, Box 1065, New York, NY 10029. E-mail: patrizia.casaccia@mssm.edu.

DOI:10.1523/JNEUROSCI.2873-15.2016

Copyright $\odot 2016$ the authors $\quad 0270-6474 / 16 / 360806-08 \$ 15.00 / 0$
}

connecting spatial constraints to transcriptional changes have not been identified. Previous work on epigenetics of developmental myelination highlighted the importance of heterochromatin formation during oligodendrocyte differentiation (Marin-Husstege et al., 2002; Ye et al., 2009; Magri et al., 2014; Liu et al., 2015), with an emphasis on histone deacetylation and repressive trimethylation of lysine residues (Marin-Husstege et al., 2002; Shen et al., 2005; Ye et al., 2009; Liu and Casaccia, 2010; Sher et al., 2012; Liu et al., 2015).

In this study we sought to identify the molecules responsible for converting mechanostimulation (i.e., response to spatial constraints in the presence of constant soluble signals) into changes of the epigenetic landscape of oligodendrocytes.

\section{Materials and Methods}

Antibodies and reagents. Reagents used in this study were as follows: methylated K9 histone 3 (H3K9me3, ab8898); phalloidin (Alexa Fluor 546 phalloidin; A22283, ThermoFisher Scientific); Deoxyribonuclease I (D12371, ThermoFisher Scientific); MBP (SMI-99, Covance); SUN1 (ab74758), SYNE1 (ab24742), emerin (ab40688), MAG (with methanol pretreatment; MAB1567, Millipore Bioscience Research Reagents), cyclic nucleotide phosphodiesterase (CNP; SMI-91R, Covance), actin (clone AC40; A4700, Sigma-Aldrich), DAPI (D1306, ThermoFisher Sci- 

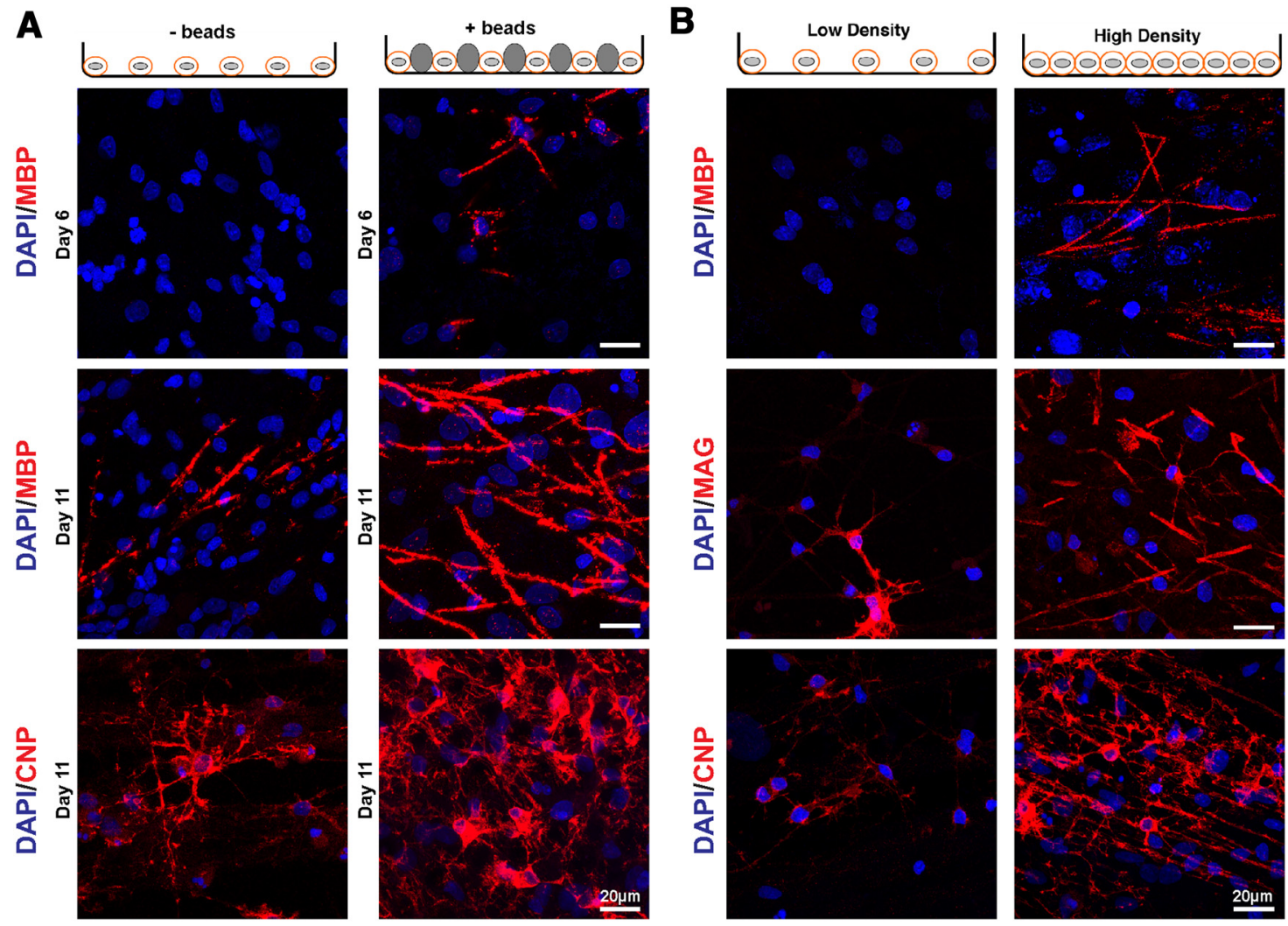

C
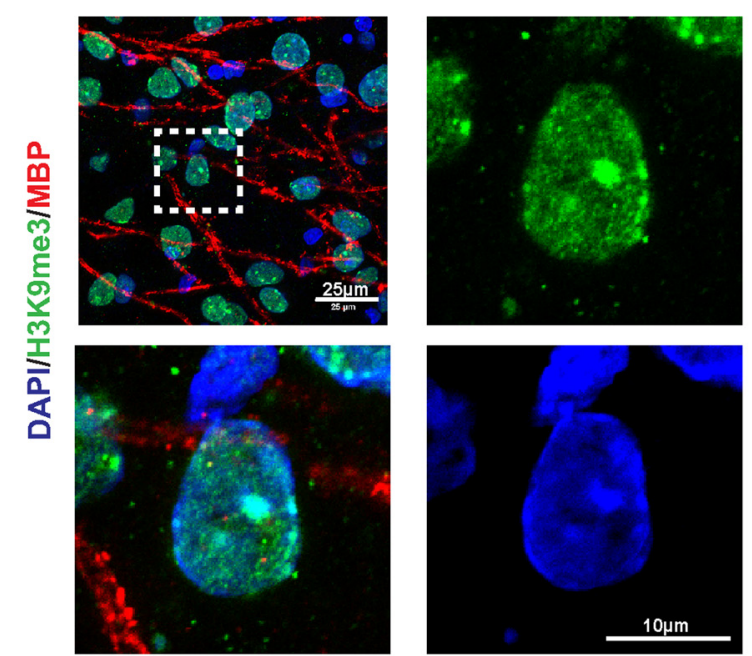

D
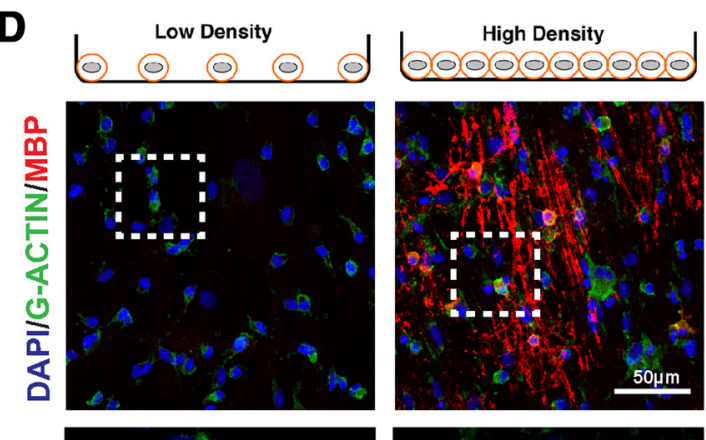

\section{$\mathbf{E}$}

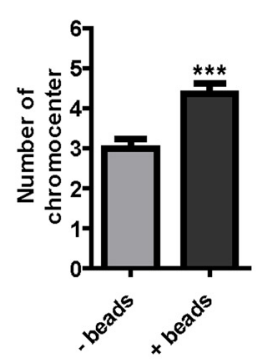

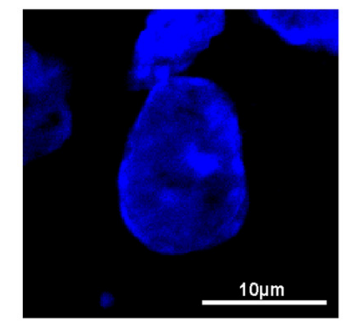

$\mathbf{F}$

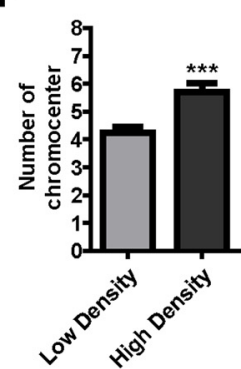

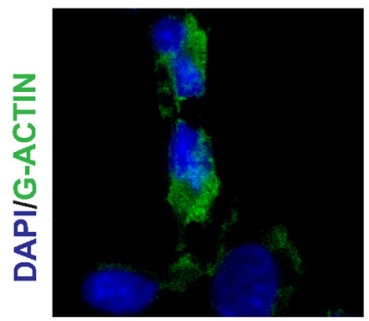

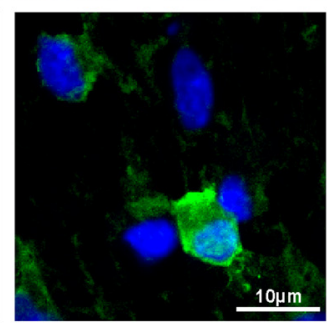

G

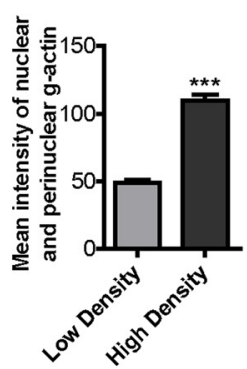

Figure 1. Nuclear changes induced by high cell density or presence of inert microspheres. $A$, Immunocytochemistry of neuronal- oligodendroglial cocultures 6 and $11 \mathrm{~d}$ after plating mouse OPCS on axons, in the absence (- beads) or presence (+ beads) of microspheres. Immunolabeling of MBP or CNP (red) to identify myelinated axons. DAPI (blue) as nuclear staining. $\boldsymbol{B}$, Neuronal-oligodendroglial coculture after plating mouse progenitors at low or high density and labeling with MBP, MAG, and CNP antibodies (red) (Figure legend continues.) 
entific). The dilutions used for these reagents were based on the manufacturer's instructions.

Animals. PDGFR $\alpha$-H2B-EGFP mice were a generous gift of Dr. Philippe Soriano (Icahn School of Medicine at Mount Sinai) and were handled in accordance with Institutional Animal Care and Use Committee-approved protocols. Mice were maintained in a pathogenfree environment at the Icahn School of Medicine at Mount Sinai animal facility. All animal procedures were approved by the Institutional Animal Care and Use Committee at Icahn School of Medicine at Mount Sinai. Timed pregnancy mice were purchased from The Jackson Laboratory.

Oligodendrocyte cultures. Oligodendrocyte progenitor cells (OPCs) were isolated from postnatal day (P) 6 male and female mice. After immunopanning for negative (BSL1 or RAN2/GC) and positive selection (PDGFRA or O4), cells were kept proliferating in PDGF $(10 \mathrm{ng} / \mathrm{ml})$ and FGF (20 ng/ml) for $5 \mathrm{~d}$. Then mitogens were removed and T3 $(30 \mathrm{ng} / \mathrm{ml})$ was added to induce differentiation into oligodendrocytes (Emery and Dugas, 2013).

DRG neuron-oligodendrocyte cocultures. Cocultures of DRG neurons and oligodendrocytes with and without microspheres were performed as described by Rosenberg et al. (2008).

Mechanostimulation using the cell-shortening device. Before plating the cells, silicone sheets ( 0.20 inch NRV G/G 40D, SMI Silicone Sheeting) were washed, sterilized with UV light, assembled into a mechanical device built in-house, stretched, and coated with Matrigel (catalog \#BD 356230 , Corning) for $30 \mathrm{~min}$ at $37^{\circ} \mathrm{C}$. As control, we used nonstretched silicon sheets on a culture plate. We seeded 250,000 cells per sheet (either stretched or not). Cells were allowed to attach at $37^{\circ} \mathrm{C}$ before adding medium to the culture plates. Mechanical stimulation was performed $24 \mathrm{~h}$ later by removing a spacer between the silicone membrane and the device, to deliver mechanical compression to the cells. Fixation was performed with $4 \%$ paraformaldehyde in phosphate buffer.

Immunocytochemistry. After blocking nonspecific staining (phosphate buffer supplemented with gelatin, bovine albumin, and $10 \%$ normal goat serum and $0.05 \%$ Triton X-100), cells were incubated with primary antibodies overnight at $4^{\circ} \mathrm{C}$, visualized by incubation with labeled secondary antibodies, and counterstained with $4^{\prime}, 6^{\prime}$-diamidino-2-phenylindole (DAPI; 1:2000) to identify nuclei. Monomeric G-actin was stained using Deoxyribonuclease I. F-actin was stained with phallodin. Labeled cells were imaged in five fields from three separate cultures using a confocal microscope (LSM710, Zeiss) or a fluorescence microscope with Apotome (Zeiss Axio Imager Z1). H3K9me3 and G-actin fluorescence staining was measured in arbitrary units, using National Institutes of Health ImageJ software.

Immunohistochemistry. For immunohistochemistry, animals were perfused with $4 \%$ paraformaldehyde. After overnight postfixation in the same solution at $4^{\circ} \mathrm{C}$, the tissue was cryopreserved in $30 \%(\mathrm{w} / \mathrm{v})$ sucrose, embedded in optimal cutting temperature compound and sectioned (12 $\mu \mathrm{m})$. Frozen sections were first permeabilized with blocking buffer $[0.1 \mathrm{M}$ phosphate buffer, $10 \%(\mathrm{v} / \mathrm{v})$ normal goat serum (Vector Laboratories), and $0.5 \%(\mathrm{v} / \mathrm{v})$ Triton X-100], followed by antigen retrieval using $10 \mathrm{~mm}$ citrate buffer, $\mathrm{pH} 6.0$, for $1 \mathrm{~h}$ at $65^{\circ} \mathrm{C}$. Incubation with primary antibodies was performed overnight at $4^{\circ} \mathrm{C}$. The next day, tissue slices were incubated with the appropriate secondary antibody for $1 \mathrm{~h}$. Monomeric G-actin was stained with Deoxyribonuclease I according to the vendor's instructions. Sections were then counterstained with DAPI $(1: 20,000)$ to visualize nuclei. Stained tissue was imaged in using a confocal microscope (LSM710, Zeiss).

$\leftarrow$

(Figure legend continued.) to identify myelinated fibers. C, High magnification of oligodendrocyte nuclei within the area delimited by dashed lines at low magnification reveals intense colabeling of the heterochromatic mark H3K9me3 (green) with DAPI-intense labeled chromocenters. $\boldsymbol{D}$, Identification of nuclear $\mathrm{G}$-actin (green) in MBP-immunoreactive oligodendrocytes (red) at high cell-density plating. $\boldsymbol{E}-\boldsymbol{F}$, Bar graphs defining chromocenter (i.e., DAPI-intense puncta) quantification in the indicated coculture conditions. $\mathbf{G}$, Bar graph of nuclear and perinuclear $\mathrm{G}$-actin as measured by fluorescent labeling quantification. At high-density plating, $\mathrm{G}$-actin was increased. Bar graphs represent average $\pm \mathrm{SEM}\left({ }^{* * *} p<0.001 ; n=\right.$ nuclei of 3 independent experiments).
Coimmunoprecipitation and Western blot. Protein extracts were obtained by lysis in $10 \mathrm{~nm}$ HEPES, $5 \mathrm{~mm}$ EDTA, $50 \mathrm{~mm} \mathrm{NaCl}, 1 \%$ Triton $\times$ 100 containing a protease inhibitor mixture (catalog \#11836153001, Roche) for $20 \mathrm{~min}$ at $4 \mathrm{C}$. Immunoprecipitation of $2 \mathrm{mg}$ of proteins was performed overnight using $2 \mu \mathrm{g}$ of the corresponding antibody. Immunoprecipitated samples and whole-cell lysates were separated by SDSPAGE and transferred to a PVDF (Millipore) membrane using a transfer buffer composed of $25 \mathrm{~mm}$ Tris base, $\mathrm{pH} 8.3,192 \mathrm{~mm}$ glycine, 20\% methanol for $75 \mathrm{~min}$ at $100 \mathrm{~V}$ at $4^{\circ} \mathrm{C}$. Western blot analysis was performed using the appropriate dilution of primary antibodies. Immunoreactive bands were visualized using horseradish peroxidase-conjugated secondary antibodies (GE Healthcare).

Quantitative real-time PCR. RNA was isolated following manufacturer's instruction and cleaned using RNeasy Mini kit (Qiagen) and amplified using the following primers for real-time PCR: for Syne1: GGGAGAGGCAGG AACGTCGC and GCGTTCGTGGCTGGAACCTG; for Sun1: TCAAG CACTGCGAAGGACTA and CTCCCTAGCCACCAGAAGGT: for GAP $D H$ : ACCCAGAAGACTGTGGATGG and CACATTGGGGGTAGG AACAC.

Lentiviral infection. Silencing of mouse primary OPCs was obtained using lentiviral transduction particles from Sigma-Aldrich (shRNAs targeting sequences for Syne1: TRCN0000239245; Emd: TRCN0000240585, and TurboGFPcontrol: SHC003V) in suspension at a multiplicity of infection of five with polybrene $(2 \mu \mathrm{g} / \mathrm{ml})$. After $2 \mathrm{~h}$ at $37^{\circ} \mathrm{C}$, cells were plated at the desired density. For cocultures, OPCs were first seeded onto $10 \mathrm{~cm}$, poly-lysine-coated dishes and incubated with shRNA lentiviruses overnight at $37^{\circ} \mathrm{C}$. Infected OPCs were then trypsinized and seeded onto DRG cultures with or without beads at the desired density.

Statistical methods. Student's $t$ test was used to determine the statistical significance between two groups of independent variables.

\section{Results}

To define the molecular players transducing spatial constraints into epigenetic signals in OPCs, we compared the effect of three distinct stimuli: high cell density, presence of inert microspheres, and application of mechanostimulation using a mechanical device in oligodendrocyte cultures. We observed that in cocultures of OPCs with neurons, inert microspheres or high-density plating of OPCs resulted in increased myelination, as demonstrated by the staining of three myelin markers: $\mathrm{MBP}, \mathrm{CNP}$, and $\mathrm{MAG}$ (Fig. 1 A, B; Rosenberg et al., 2008). Myelination was also accompanied by changes in nuclear structure, characterized by the presence of H3K9me3-immunoreactive heterochromatin (Fig. 1C) and a higher number of chromocenters (DAPI puncta underneath the nuclear membrane) in oligodendrocyte nuclei (Fig. 1E,F). Interestingly, we observed that G-actin subcellular distribution was also influenced by cell density. Nuclear and perinuclear G-actin was significantly increased in high-density conditions (Fig. $1 D, G$ ) and this was consistent with the redistribution of actin previously described in other cell types (Maniotis et al., 1997; Hirata et al., 2014; Li et al., 2014; Nagayama et al., 2014).

To directly address the effect of mechanical forces on nuclear heterochromatin in OPCs, we also plated them on a Matrigelcoated prestretched silicone substrate, mounted on a portable device (Fig. $2 A$ ) with the ability to impose uniaxial cell deformation upon mechanical release of the substrate (Costa et al., 2002). The mechanical stimulation resulted in the instantaneous transitioning of the cells from a spread state to a compressed configuration, which was accompanied by the reorganization of nuclear structure, as shown by the number of peripheral chromocenters (Fig. 2C) and the increased nuclear/perinuclear G-actin (Fig. 2B). Therefore, acute application of mechanical force to OPCs was sufficient to redistribute actin and transduce a signal to the nuclear compartment.

A potential connection between cell membrane and nuclear components is provided by components of the linker of nucle- 
A
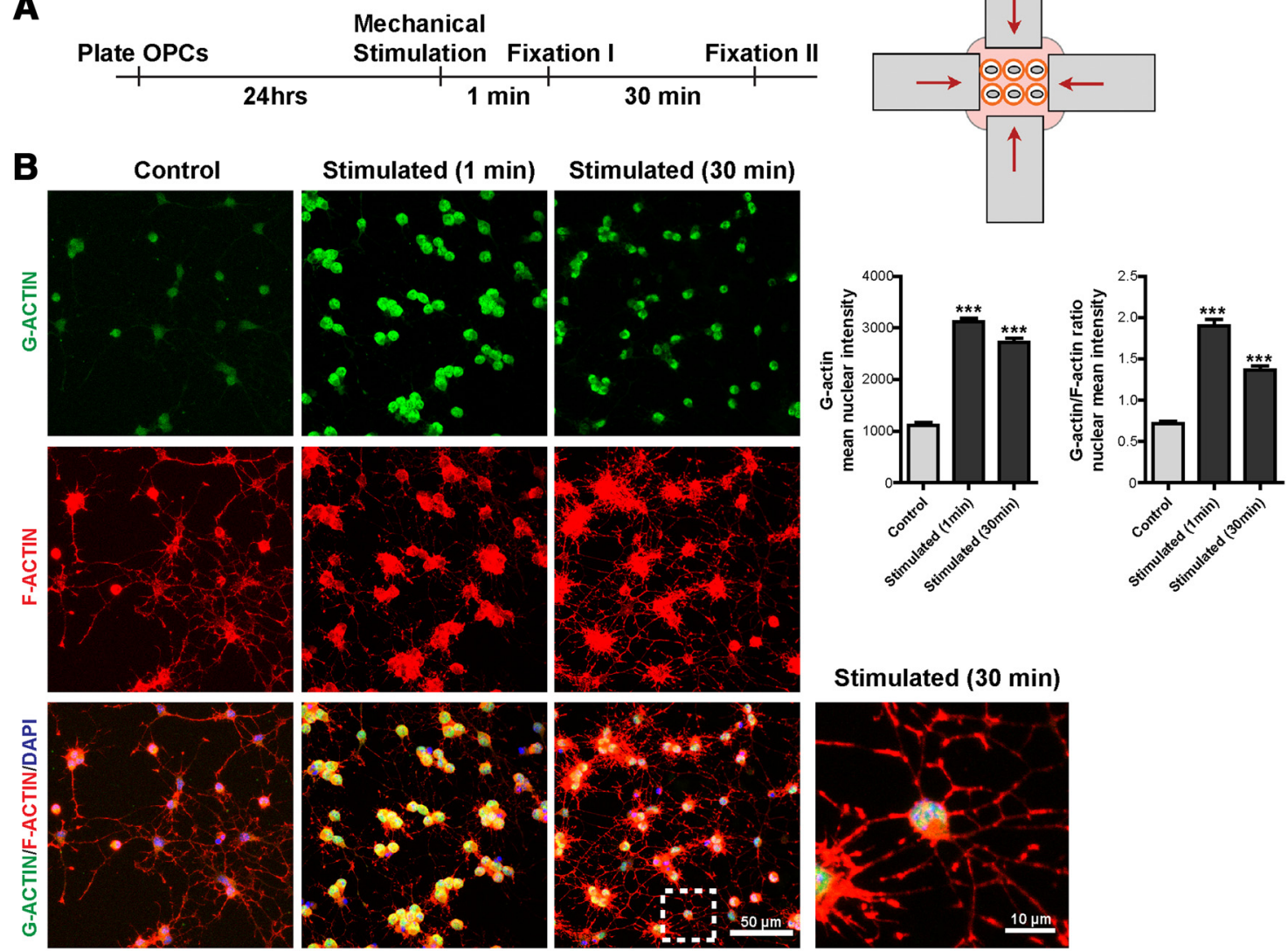

\section{Stimulated (30 $\mathrm{min})$}
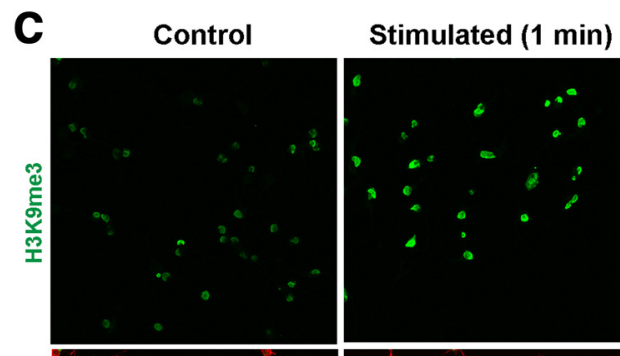

Stimulated (30 min)
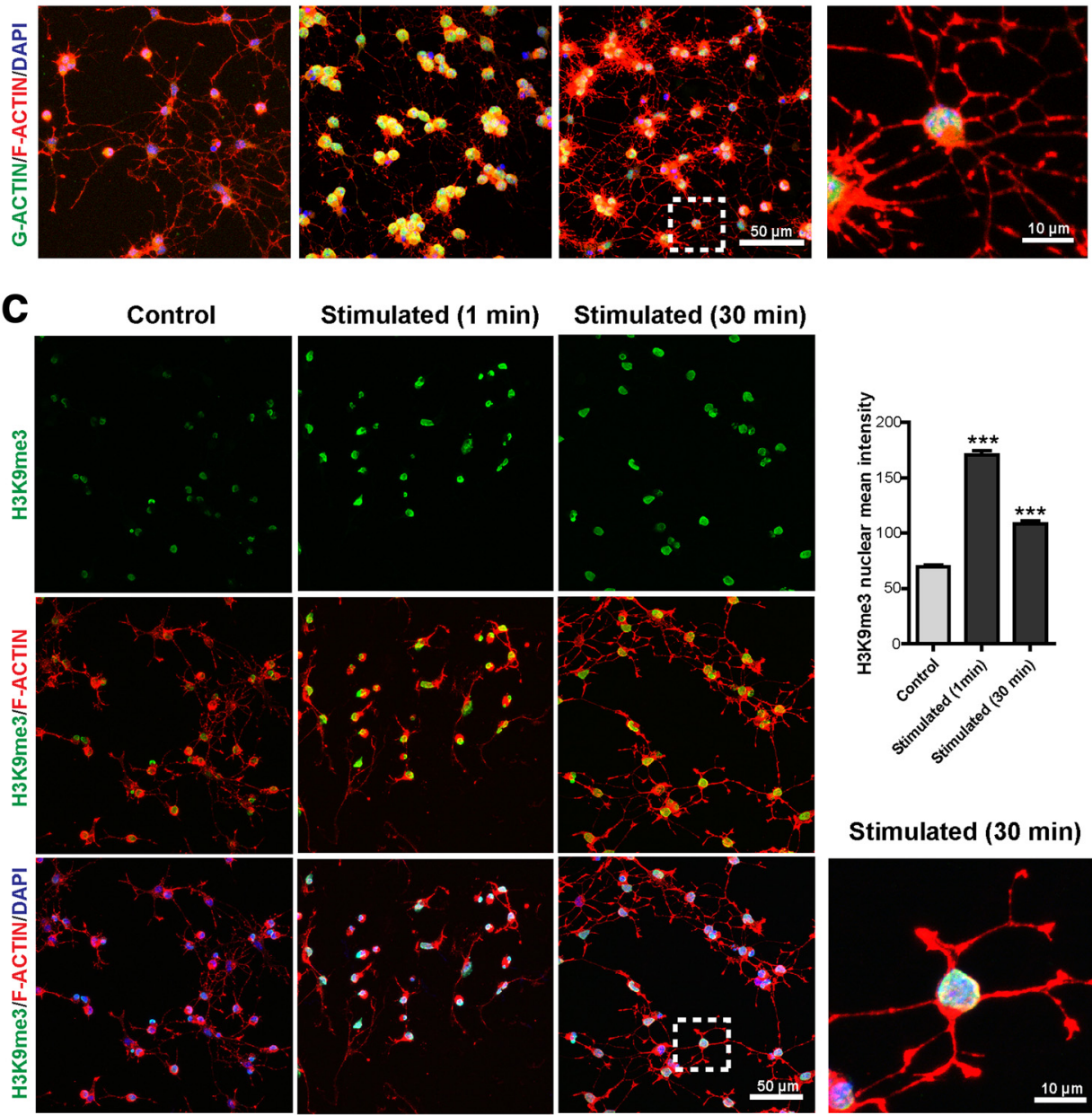

\section{Stimulated (30 $\mathrm{min})$}

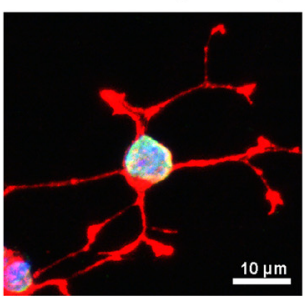

Figure 2. Mechanical forces applied to cultured progenitors modify their nuclear organization. $A$, A portable "cell-compression" device was used to apply mechanical force on cultured mouse OPCS. Cells were fixed 1 or 30 min after mechanical stimulation. B, Immunocytochemistry of stimulated and nonstimulated oligodendrocytes stained for G-actin (green) and F-actin (red). Note the increased nuclear $\mathrm{G}$-actin signal after stimulation. Quantifications are shown for $\mathrm{G}$-actin alone and for a $\mathrm{G}$-actin/F-actin ratio. The high-magnification inset reveals the healthy state of a cell after mechanostimulation. C, Immunocytochemistry of stimulated and nonstimulated oligodendrocytes stained for H3K9me3 (green), F-actin (red), and DAPI (blue). The high-magnification inset reveals the healthy state of a cell after mechanostimulation. Bar graphs represent average $\pm \operatorname{SEM}\left({ }^{* * *} p<0.001 ; n=\right.$ nuclei of 3 independent experiments). 


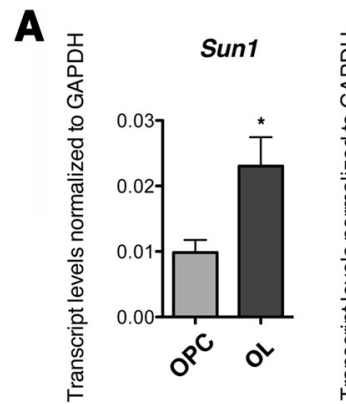

D

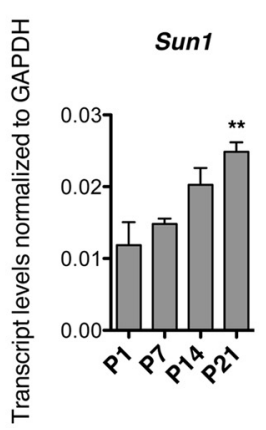

B

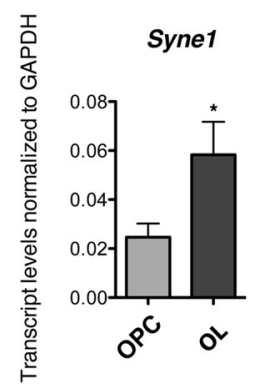

C

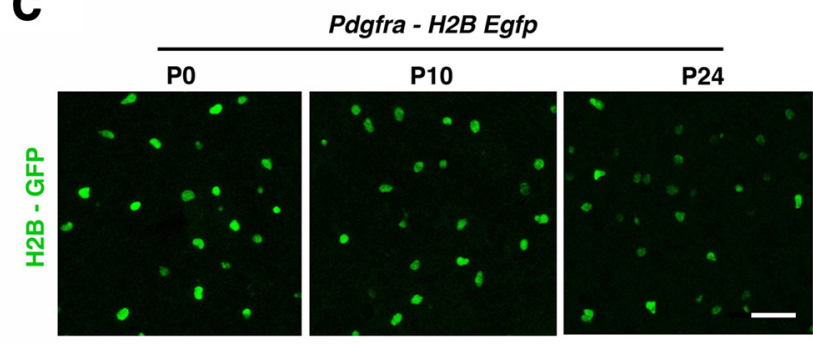

$\mathbf{E}$

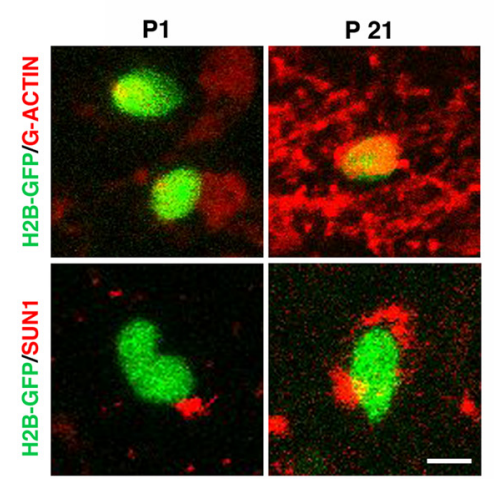

$\mathbf{F}$

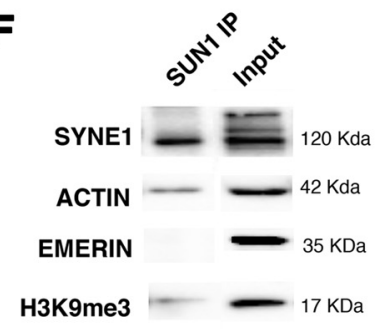

Figure 3. Transcript and protein levels of LINC complex components increase with differentiation. $\boldsymbol{A}$, Transcript levels of Sun 1 and Syne 1 measured by real-time PCR in cultured undifferentiated progenitors (OPC) as well as mature oligodendrocytes (OL). Sun1 and Syne 1 transcription increased with differentiation. $\boldsymbol{B}$, Western blot of protein extracts from progenitors and oligodendrocytes probed with antibodies specific for SUN1, SYNE1, and actin as loading control. C, Confocal images of sagittal brain sections from mice expressing H2B-EGFP under the control of the Pdgfra promoter. Positive cells in the corpus callosum at P0, P10, and P24 are shown. Scale bar, $30 \mu \mathrm{m}$. D, Transcript levels of Sun 1 and Syne 1 measured by real-time PCR in samples of RNA isolated from the developing corpus callosum at P1, P7, P14, and P21. E, Immunohistochemical analysis of the corpus callosum in Pdgfra-H2B-EGFP mice stained for G-actin (red, top) or SUN1 (red, bottom) at P1 and P21. Scale bar, $5 \mu \mathrm{m}$.F, Immunoprecipitation of SUN1 from spinal cord extracts of P21 mice, followed by Western blot analysis revealed the formation of a protein complex with SYNE1, actin, and H3K9me3, but not with emerin (error bars: SEM, $n=3$ independent experiments; ${ }^{*} p<0.05,{ }^{* *} p<0.01,{ }^{* * *} p<0.001$ ).

oskeleton and cytoskeleton (LINC) complex (Anno et al., 2012; Martins et al., 2012; Jahed et al., 2014; Kaminski et al., 2014), which is composed of SUN (Sad1, UNC-84) and KASH (Klarsicht, ANC-1, and Syne homology) domain-containing proteins (Starr and Fischer, 2005; Razafsky and Hodzic, 2009; Sosa et al., 2012). The expression of Sun1 and Syne1 was first evaluated during the differentiation of OPCs into oligodendrocytes in vitro, which revealed increased transcripts and protein levels (Fig. $3 \mathrm{~A}$, $B$ ). Similar results were obtained in vivo in the corpus callosum of mice expressing H2B-EGFP under the control of the Pdgfra promoter (Fig. $3 C, D$ ). Because SYNE1 has the ability to bind actin with its large $\mathrm{N}$-terminal domain and to bind the nuclear protein SUN1 with its C-terminal domain (Ostlund et al., 2009), we conducted immunohistochemistry of the developing corpus callosum, using antibodies specific for SUN1 and actin at two developmental time points before (i.e., P1) and during myelination (i.e., P21; Fig. 3E). In agreement with the involvement of LINC complexes in myelination, nuclear colocalization of actin and SUN1 was only detected at P21 (Fig. 3E). Consistent with the immunohistochemical data, we detected the formation of the LINC complex in protein extracts from the CNS of P21 mice, which, in addition to the interaction between SYNE1 and SUN1 and actin, also revealed binding with nucleosomal histones characterized by the H3K9me3 mark (Fig. 3F). To further assess the specificity of complex detection, we showed that protein extracts immunoprecipitated with anti-SUN antibodies did not bind the unrelated nuclear protein emerin (Fig. $3 F$ ). Together these results identify the LINC complex as a potential mediator of mechanoinduced heterochromatin formation in oligodendrocytes during myelination. This hypothesis was tested by silencing Syne1 using lentiviral vectors and evaluating the response of
OPCs to mechanostimulation induced by the device. Decreased Syne1 levels in OPCs resulted in impaired heterochromatin (Fig. $4 A$ ) and in chromocenter formation (Fig. 4B) and aberrant subcellular distribution of G-actin after mechanical stimulation (Fig. $4 C)$. To further define whether pathways transducing spatial information were independent of those activated by chemical signals, we also assessed the ability of Syne1-silenced OPCs to differentiate in response to T3 thyroid hormone treatment and compared these results to those obtained in the presence of artificial microspheres. Syne1 silencing had no effect on T3-induced differentiation (Fig. 4D) while it profoundly affected the prodifferentiative effect of microsphere plating (Fig. $4 E$ ). The effect was specific for components of the LINC complex, since silencing the nonrelated nuclear envelope protein emerin (Emd) did not affect the number of myelinated fibers (Fig. $4 D, E$ ). We conclude that space limitations in OPCs promote heterochromatin formation and differentiation by activating a pathway involving the formation of SYNE-SUN-actin complexes.

\section{Discussion}

OPCs respond to conditions associated with limited space, as induced by plating of microspheres (Rosenberg et al., 2008) with enhanced differentiation and increased myelinated fibers in coculture. They can also respond to cell density by changing nuclear shape, and to stiffness of the extracellular matrix (Jagielska et al., 2012) by readjusting migration and differentiation rates. While the evidence linking physical stimulation to functional effect is clear, the molecules responsible for transducing the effect of an external force into a program of differentiation remain elusive.

In this study we have shown that distinct modalities of compressive force applied onto cultured OPCs favor their differ- 
A Non-stimulated

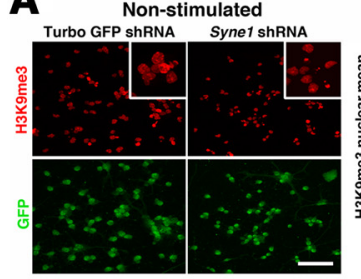

Stimulated (30 $\mathrm{min})$

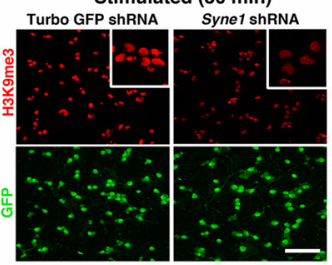

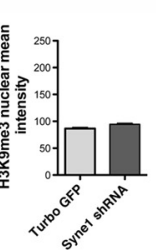

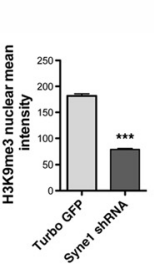

B

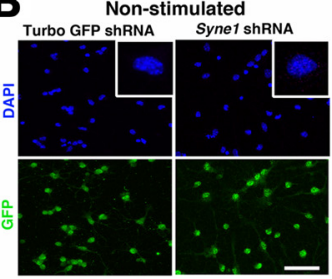

Stimulated (30 $\mathrm{min})$

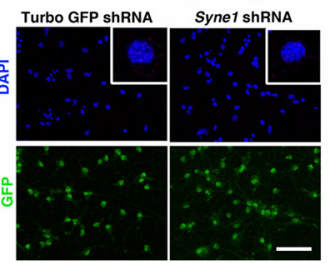

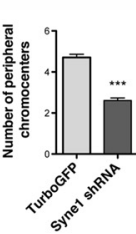

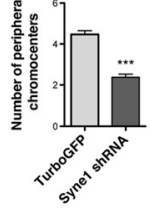

C

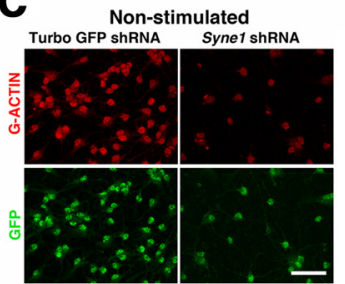

Stimulated (30 min)

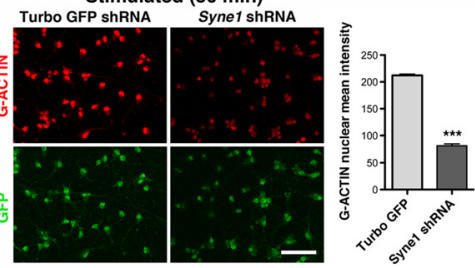

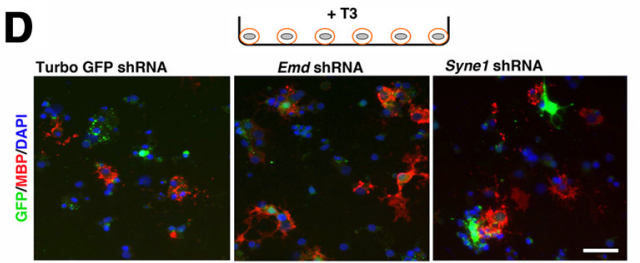
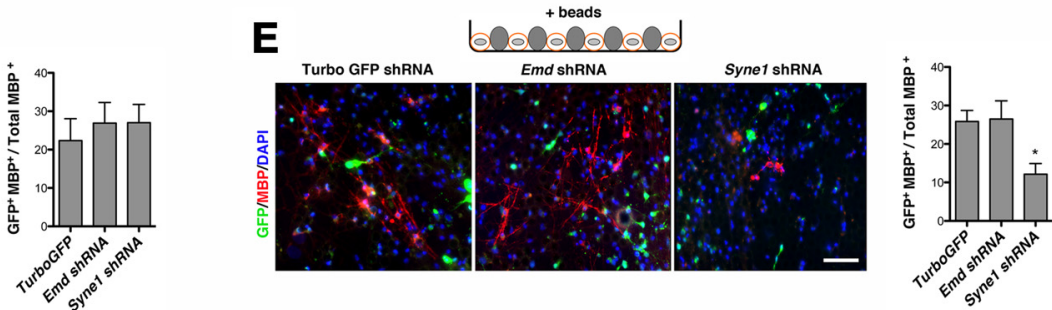

Figure 4. Syne1 silencing prevents the effect of mechanostimulation on oligodendrocytes. $A-C$, Confocal images of cultured mouse oligodendrocyte progenitors infected with Turbo GFP control or Syne1 shRNA lentiviruses identified by green fluorescence due to EGFP ( $\boldsymbol{A}-\boldsymbol{C}$, green) and immunolabeled by H3K9me3 antibodies ( $\boldsymbol{A}$, red), by DAPl as nuclear staining ( $\boldsymbol{B}$, blue), by $\mathrm{G}$-actin-specific antibodies ( $\boldsymbol{C}$, red). Note that silencing of Syne 1 precluded the nuclear changes induced by mechanostimulation. $\boldsymbol{D}-\boldsymbol{E}$, Oligodendrocyte progenitors were infected with Turbo GFP control, Emd shRNA lentiviruses, or Syne1 shRNA lentiviruses before inducing differentiation with T3 (D) or plating them on neurons in the presence of microspheres (E). Silencing of Syne 1 did not affect MBP expression induced by chemical signals (i.e., T3 treatment; $\boldsymbol{D})$, but decreased MBP expression induced by mechanostimulation caused by the presence of microspheres $(\boldsymbol{E},+$ beads). Bar graphs represent average $\pm \operatorname{SEM}\left({ }^{*} p<0.05,{ }^{* *} p<0.01,{ }^{* * *} p<0.001 ; n=3\right.$ independent experiments). Scale bar, $50 \mu \mathrm{m}$.

entiation into myelinating oligodendrocytes. In addition to highdensity plating, compressive forces were generated either by interspersing inert microspheres with cultured OPCs or by using a mechanical device. All these conditions resulted in the formation of nuclear heterochromatin, a hallmark of differentiation and increased myelination. Because the nuclear structure dynamic has been recently shown to modulate transcription, DNA replication and repair, and RNA processing (Schneider and Grosschedl, 2007), we reasoned that the rearrangements induced by compressive forces would be sufficient to initiate a differentiation program.

We have highlighted the existence of a cytosolic connectionmediating force transmission from the plasma membrane onto the nucleus, demonstrated by the redistribution of G-actin to the nuclear/perinuclear space only in stimulated cells, revealing the possibility that actin dynamics in regions with greater mechanical stress could modulate the mechanotransduction pathway. It has been recently shown that F-actin depolymerization is critical for myelination and necessary for the formation of myelin wraps along the axons in the CNS (Nawaz et al., 2015; Zuchero et al., 2015). However, the concept of G-actin-mediated modulation of a transcriptional program accompanying oligodendrocyte morphological changes has not been reported. In this context, our study identifies SYNE1, a component of the LINC complex, as an important molecular link coupling cytoskeletal changes with heterochromatin formation in cultured cells.

Because all the different modalities of mechanostimulation assessed in this study resulted in increased heterochromatin, as shown by the detection of repressive histone marks and chromocenters, it is remarkable to identify the KASH domain- containing protein SYNE1 as a key signaling molecule for mechanotransduction in oligodendrocytes. The ability of SYNE1's N-terminal cytosolic domain to interact with cytosolic actin and of its C-terminal domain to interact with the nuclear periphery (Lombardi et al., 2011) and with nucleosomal histone carrying repressive marks further highlight SYNE1's importance in transducing a mechanical signal into nuclear changes with likely transcriptional consequences. Although the identity of the genes affected by these repressive marks is not part of this study, it is conceivable that oligodendrocytes might rely on mechanosensitive transcription factors as reported in other cell types (Mammoto et al., 2012; Wang et al., 2015).

Decreased myelination was only detected in Syne1-silenced OPCs that were induced to differentiate by biophysical constraints (i.e., plating with microspheres), while differentiation induced by mitogen withdrawal and T3 supplementation were unaffected, thereby revealing the specificity of the SYNE-SUNactin complex for mechanotransduction.

In conclusion, our study identifies signaling molecules relating spatial constraints to the activation of oligodendrocyte differentiation. Our results reveal SYNE1 as a key mediator of heterochromatin formation and differentiation induced by mechanical stimuli. Based on its distinct binding domain, SYNE1 has the ability to "sense" actin dynamics in the cytoplasm and "transfer" the signal to the inner nuclear membrane and chromatin, thereby linking external forces of compression to epigenetic changes associated with heterochromatin formation. We have previously shown that removal of mitogens from the culture medium also induces heterochromatin for- 
mation (Liu et al., 2015) but that pathways of "chemically induced" differentiation do not rely on SYNE1 function. Therefore, OPCs respond to multiple modalities of differentiation by activating distinct signaling pathways to integrate spatial and chemical signals and induce heterochromatin formation and oligodendrocyte identity.

Because the viscoelasticity of the brain has been documented to change in response to demyelinating lesions (Schregel et al., 2012) and because OPCs are mechanosensitive (Jagielska et al., 2012), a thorough analysis of mechanotransduction signals in conditions of failed remyelination is worth considering. This study, together with previous reports, further highlights the complexity of remyelination and suggests that a combination of mechanical and chemical stimuli might contribute to the optimization of therapeutic approaches and strategies to address demyelinating conditions and promote remyelination.

\section{References}

Anno T, Sakamoto N, Sato M (2012) Role of nesprin-1 in nuclear deformation in endothelial cells under static and uniaxial stretching conditions. Biochem Biophys Res Commun 424:94-99. CrossRef Medline

Barres BA, Lazar MA, Raff MC (1994) A novel role for thyroid hormone, glucocorticoids and retinoic acid in timing oligodendrocyte development. Development 120:1097-1108. Medline

Costa KD, Hucker WJ, Yin FC (2002) Buckling of actin stress fibers: a new wrinkle in the cytoskeletal tapestry. Cell Motil Cytoskeleton 52:266-274. CrossRef Medline

Dugas JC, Ibrahim A, Barres BA (2012) The T3-induced gene KLF9 regulates oligodendrocyte differentiation and myelin regeneration. Mol Cell Neurosci 50:45-57. CrossRef Medline

Emery B, Dugas JC (2013) Purification of oligodendrocyte lineage cells from mouse cortices by immunopanning. Cold Spring Harb Protoc 2013: 854-868. Medline

Fortin D, Rom E, Sun H, Yayon A, Bansal R (2005) Distinct fibroblast growth factor (FGF)/FGF receptor signaling pairs initiate diverse cellular responses in the oligodendrocyte lineage. J Neurosci 25:7470-7479. CrossRef Medline

Fünfschilling U, Supplie LM, Mahad D, Boretius S, Saab AS, Edgar J, Brinkmann BG, Kassmann CM, Tzvetanova ID, Möbius W, Diaz F, Meijer D, Suter U, Hamprecht B, Sereda MW, Moraes CT, Frahm J, Goebbels S, Nave KA (2012) Glycolytic oligodendrocytes maintain myelin and longterm axonal integrity. Nature 485:517-521. Medline

Furusho M, Kaga Y, Ishii A, Hébert JM, Bansal R (2011) Fibroblast growth factor signaling is required for the generation of oligodendrocyte progenitors from the embryonic forebrain. J Neurosci 31:5055-5066. CrossRef Medline

Gao FB, Apperly J, RaffM (1998) Cell-intrinsic timers and thyroid hormone regulate the probability of cell-cycle withdrawal and differentiation of oligodendrocyte precursor cells. Dev Biol 197:54-66. CrossRef Medline

Hart IK, Richardson WD, Bolsover SR, Raff MC (1989) PDGF and intracellular signaling in the timing of oligodendrocyte differentiation. J Cell Biol 109:3411-3417. CrossRef Medline

Hirata H, Sokabe M, Lim CT (2014) Molecular mechanisms underlying the force-dependent regulation of actin-to-ECM linkage at the focal adhesions. Prog Mol Biol Transl Sci 126:135-154. CrossRef Medline

Jagielska A, Norman AL, Whyte G, Vliet KJ, Guck J, Franklin RJ (2012) Mechanical environment modulates biological properties of oligodendrocyte progenitor cells. Stem Cells Dev 21:2905-2914. CrossRef Medline

Jahed Z, Shams H, Mehrbod M, Mofrad MR (2014) Mechanotransduction pathways linking the extracellular matrix to the nucleus. Int Rev Cell Mol Biol 310:171-220. CrossRef Medline

Kaminski A, Fedorchak GR, Lammerding J (2014) The cellular mastermind(?)-mechanotransduction and the nucleus. Prog Mol Biol Transl Sci 126:157-203. CrossRef Medline

Lee Y, Morrison BM, Li Y, Lengacher S, Farah MH, Hoffman PN, Liu Y, Tsingalia A, Jin L, Zhang PW, Pellerin L, Magistretti PJ, Rothstein JD (2012) Oligodendroglia metabolically support axons and contribute to neurodegeneration. Nature 487:443-448. CrossRef Medline

Li Q, Kumar A, Makhija E, Shivashankar GV (2014) The regulation of dy- namic mechanical coupling between actin cytoskeleton and nucleus by matrix geometry. Biomaterials 35:961-969. CrossRef Medline

Liu J, Casaccia P (2010) Epigenetic regulation of oligodendrocyte identity. Trends Neurosci 33:193-201. CrossRef Medline

Liu J, Magri L, Zhang F, Marsh NO, Albrecht S, Huynh JL, Kaur J, Kuhlmann T, Zhang W, Slesinger PA, Casaccia P (2015) Chromatin landscape defined by repressive histone methylation during oligodendrocyte differentiation. J Neurosci 35:352-365. CrossRef Medline

Lombardi ML, Jaalouk DE, Shanahan CM, Burke B, Roux KJ, Lammerding J (2011) The interaction between nesprins and sun proteins at the nuclear envelope is critical for force transmission between the nucleus and cytoskeleton. J Biol Chem 286:26743-26753. CrossRef Medline

Lu QR, Yuk D, Alberta JA, Zhu Z, Pawlitzky I, Chan J, McMahon AP, Stiles CD, Rowitch DH (2000) Sonic hedgehog-regulated oligodendrocyte lineage genes encoding bHLH proteins in the mammalian central nervous system. Neuron 25:317-329. CrossRef Medline

Magri L, Swiss VA, Jablonska B, Lei L, Pedre X, Walsh M, Zhang W, Gallo V, Canoll P, Casaccia P (2014) E2F1 coregulates cell cycle genes and chromatin components during the transition of oligodendrocyte progenitors from proliferation to differentiation. J Neurosci 34:1481-1493. CrossRef Medline

Mammoto A, Mammoto T, Ingber DE (2012) Mechanosensitive mechanisms in transcriptional regulation. J Cell Sci 125:3061-3073. CrossRef Medline

Maniotis AJ, Chen CS, Ingber DE (1997) Demonstration of mechanical connections between integrins, cytoskeletal filaments, and nucleoplasm that stabilize nuclear structure. Proc Natl Acad Sci U S A 94:849-854. CrossRef Medline

Marin-Husstege M, Muggironi M, Liu A, Casaccia-Bonnefil P (2002) Histone deacetylase activity is necessary for oligodendrocyte lineage progression. J Neurosci 22:10333-10345. Medline

Martins RP, Finan JD, Guilak F, Lee DA (2012) Mechanical regulation of nuclear structure and function. Annu Rev Biomed Eng 14:431-455. CrossRef Medline

Nagayama K, Yamazaki S, Yahiro Y, Matsumoto T (2014) Estimation of the mechanical connection between apical stress fibers and the nucleus in vascular smooth muscle cells cultured on a substrate. J Biomech 47: 1422-1429. CrossRef Medline

Nawaz S, Sánchez P, Schmitt S, Snaidero N, Mitkovski M, Velte C, Brückner BR, Alexopoulos I, Czopka T, Jung SY, Rhee JS, Janshoff A, Witke W, Schaap IA, Lyons DA, Simons M (2015) Actin filament turnover drives leading edge growth during myelin sheath formation in the central nervous system. Dev Cell 34:139-151. CrossRef Medline

Nery S, Wichterle H, Fishell G (2001) Sonic hedgehog contributes to oligodendrocyte specification in the mammalian forebrain. Development 128: 527-540. Medline

Ostlund C, Folker ES, Choi JC, Gomes ER, Gundersen GG, Worman H] (2009) Dynamics and molecular interactions of linker of nucleoskeleton and cytoskeleton (LINC) complex proteins. J Cell Sci 122:4099-4108. CrossRef Medline

Pombo PM, Barettino D, Ibarrola N, Vega S, Rodríguez-Pena A (1999) Stimulation of the myelin basic protein gene expression by 9 -cis-retinoic acid and thyroid hormone: activation in the context of its native promoter. Brain Res Mol Brain Res 64:92-100. CrossRef Medline

Razafsky D, Hodzic D (2009) Bringing KASH under the SUN: the many faces of nucleo-cytoskeletal connections. J Cell Biol 186:461-472. CrossRef Medline

Rinholm JE, Hamilton NB, Kessaris N, Richardson WD, Bergersen LH, Attwell D (2011) Regulation of oligodendrocyte development and myelination by glucose and lactate. J Neurosci 31:538-548. CrossRef Medline

Rosenberg SS, Kelland EE, Tokar E, De la Torre AR, Chan JR (2008) The geometric and spatial constraints of the microenvironment induce oligodendrocyte differentiation. Proc Natl Acad Sci U S A 105:14662-14667. CrossRef Medline

Samanta J, Kessler JA (2004) Interactions between ID and OLIG proteins mediate the inhibitory effects of BMP4 on oligodendroglial differentiation. Development 131:4131-4142. CrossRef Medline

Schneider R, Grosschedl R (2007) Dynamics and interplay of nuclear architecture, genome organization, and gene expression. Genes Dev 21: 3027-3043. CrossRef Medline

Schregel K, Wuerfel E, Garteiser P, Gemeinhardt I, Prozorovski T, Aktas O, Merz H, Petersen D, Wuerfel J, Sinkus R (2012) Demyelination reduces 
brain parenchymal stiffness quantified in vivo by magnetic resonance elastography. Proc Natl Acad Sci U S A 109:6650-6655. CrossRef Medline

Shen S, Li J, Casaccia-Bonnefil P (2005) Histone modifications affect timing of oligodendrocyte progenitor differentiation in the developing rat brain. J Cell Biol 169:577-589. CrossRef Medline

Sher F, Boddeke E, Olah M, Copray S (2012) Dynamic changes in Ezh2 gene occupancy underlie its involvement in neural stem cell self-renewal and differentiation towards oligodendrocytes. PLoS One 7:e40399. CrossRef Medline

Sosa BA, Rothballer A, Kutay U, Schwartz TU (2012) LINC complexes form by binding of three KASH peptides to domain interfaces of trimeric SUN proteins. Cell 149:1035-1047. CrossRef Medline

Starr DA, Fischer JA (2005) KASH ' $n$ Karry: the KASH domain family of cargo-specific cytoskeletal adaptor proteins. Bioessays 27:1136-1146. CrossRef Medline

Wang J, Zhang Y, Zhang N, Wang C, Herrler T, Li Q (2015) An updated review of mechanotransduction in skin disorders: transcriptional regulators, ion channels, and microRNAs. Cell Mol Life Sci 72:2091-2106. CrossRef Medline
Wu M, Hernandez M, Shen S, Sabo JK, Kelkar D, Wang J, O’Leary R, Phillips GR, Cate HS, Casaccia P (2012) Differential modulation of the oligodendrocyte transcriptome by sonic hedgehog and bone morphogenetic protein 4 via opposing effects on histone acetylation. J Neurosci 32:66516664. CrossRef Medline

Ye F, Chen Y, Hoang T, Montgomery RL, Zhao XH, Bu H, Hu T, Taketo MM, van Es JH, Clevers H, Hsieh J, Bassel-Duby R, Olson EN, Lu QR (2009) HDAC1 and HDAC2 regulate oligodendrocyte differentiation by disrupting the beta-catenin-TCF interaction. Nat Neurosci 12:829-838. CrossRef Medline

Zhou YX, Armstrong RC (2007) Interaction of fibroblast growth factor 2 (FGF2) and notch signaling components in inhibition of oligodendrocyte progenitor (OP) differentiation. Neurosci Lett 421:27-32. CrossRef Medline

Zuchero JB, Fu MM, Sloan SA, Ibrahim A, Olson A, Zaremba A, Dugas JC, Wienbar S, Caprariello AV, Kantor C, Leonoudakus D, LariosaWillingham K, Kronenberg G, Gertz K, Soderling SH, Miller RH, Barres BA (2015) CNS myelin wrapping is driven by actin disassembly. Dev Cell 34:152-167. CrossRef Medline 